\title{
The Effect of âduOn-Lineâu( POCT on Patient waiting times in an Accident and Emergency Department
}

\author{
Ashfaq Gilkar, Richard Fink, Philip Eardley, Catriona Barron
}

\begin{abstract}
This POCT (point of care testing) team was constructed at the start of 2012 to implement the POCT project aiming to define and test the hypothesis that interfacing POCT devices to a clinical electronic order communications system reduces patient waiting times in an NHS Accident and Emergency Department (A\&E). The devices selected for evaluation initially comprised the Sysmex XS 1000i haematology analyser and the Abbott i-Stat chemistry analyser.
\end{abstract}

The POCT devices were interfaced to a server (Midlynx) which in turn was connected via the hospital internal network, to the ICE system (Integrated Clinical Environment). Test orders entered on the ICE system produced a bar code label read by the individual POCT devices. Once required tests were assayed, the results were transmitted back to the ICE system, where they could be viewed by users across the hospital site. The quality of POCT analytical performance was assessed by running quality control checks as recommended by the manufacturers and by exchanging samples daily with the clinical laboratory. The I-Stat (a Chemistry analyser manufactured by 'Abbott plc') was also tested against material obtained from a national external quality assurance scheme (NEQAS).

The time taken to produce POCT tests was calculated as the time elapsed (TE) between requesting tests, and the time at which completed results were returned to the ICE system. Patient waiting times were derived from the patient administration system (Symphony) used in the A\&E department. To assess the true effect of POCT on patient waiting times the analysis was confined to cases associated with POCT tests only $(n=217)$. A control population $(n=229)$ was randomly selected from the clinical laboratory database. The time interval between requesting a test and receiving the results for the POCT tests was 23 minutes and for the laboratory tests, 60 minutes. The patient waiting times (time of discharge âdu time of arrival) was 167 minutes for the POCT group and 208 for the clinical laboratory group, a difference of 31 minutes. The project confirmed that interfaced (on-line) POCT has many advantages with respect to the quality and safety of data management. The benefits include reliable identification of the requesting clinician and patient and the assurance that the right result is allocated to the right patient. The study also showed that the quality of results produced by the POCT devices met the requirements of the clinical environment.

\section{Problem}

In the UK POCT (point of care testing) devices lead a lonely, solitary existence. Unlike laboratory analysers, they are not connected to networks with the result that overall efficiency and data integrity is poor. This initiative was carried out to ascertain whether interfacing POCT devices to a clinical order communications system (Sunquest ICE) improves clinical data integrity and reduces patient waiting times in an Accident and Emergency Department.

Interfacing POCT to an order communications system, rather than to a laboratory or proprietary system, (both rare events) is an unusual and innovative approach, providing clinicians with direct electronic access to diagnostic devices. This initiative principally addresses the national problem of patient waiting times in A\&E while at the same time addressing the need to improve the level of clinical data integrity in the context of conventional POCT implementations. In most diagnostic systems, the handling data adheres to recommended practice involving the registration of authenticated patient demographics, user details and the electronic rather than manual transcription of patient test results. In contrast,
POCT devices used as 'stand alone' devices are fairly promiscuous about data, accepting 'XXXX' instead of unique patient identifiers and '\#\#\#' or shared passwords in the place of pre-registered and trained users. Patient data may be printed out on paper strips and the results transcribed ( $6 \%$ error rate) into case notes.

\section{Background}

Initially, in 2010, we carried out a feasibility study of interfaced point of care testing in general practice (The Brentford Group Practice âtu Dr Stuart LaneâuDs practice). The devices measured chemistry and haematology profiles and were connected by small dedicated server to a patient electronic order communications system (ICE âDC Integrated clinical environment) manufactured by Sunquest Inc. This study proved the concept that interfaced POCT was feasible, very easy to use, reliable and likely to improve patient management in situations where the rapid availability of results was an important consideration.

In view of these positive results, the present study beginning in 2012 was designed to test whether âCDon-lineâDu POCT analysers could work in the demanding environment of an Accident 
and Emergency (A\&E) department. The objective of this study was to demonstrate whether this configuration could reduce the duration of patient waiting times and whether the interface would work reliably in the demanding environment of an A\&E department that managed over 100000 patients per year.. In the WMUH for example, the recent introduction of POCT in the anticoagulation clinic has improved the patient experience (patient surveys), reduced cost and markedly diminished waiting times. This case study shows that POCT when focused on a discrete group of patients can be very advantageous. The problem with $A \& E$, is the unpredictable arrival of patients with a large range of disorders requiring a large repertoire of different tests.

Previous studies using POCT devices in A\&E departments have shown reduced patient waiting times in some cases but not in others. Lee-Lewandrowski et al (1) measured blood glucose, urine chorionic gonadotrophin, urine dipstick, creatine kinase-MB and Toponin levels in an emergency department by POCT. and observed an overall reduction in patient length of stay of 41.3 minutes (1). Similarly, Murray et al (2) reported that tests processed by POCT compared to the central laboratory resulted in waiting times of 3 hours 28 minutes and 4 hours 22 minutes respectively. In contrast, Parvin et al (3) observed no change in the patient waiting time in a 5 week period involving 2067 patients when a hand-held device was used to estimate $\mathrm{Na}, \mathrm{K}, \mathrm{CL}$, urea and glucose concentrations. In this study, the length of stay during the POCT period was 209 minutes compared to 201 minutes for the control periods when the central laboratory was used (3).

Kendall, Reeves and Clancy (4) studying 1728 patients noted that clinical decisions were made 74 minutes earlier when POCT rather than the central laboratory was used for haematological tests; 86 minutes earlier for biochemical tests and 21 minutes earlier for blood gases. Despite these accelerated times, there was no difference between the groups in the amount of time spent in the A\&E department.

To test whether a more comprehensive repertoire of laboratory tests could influence patient waiting times, Leman et al (5) established a satellite laboratory within the boundaries of an A\&E department. Tests included electrolytes, urea, creatinine, LFT, Amylase, calcium and phosphate, $\mathrm{C}$ reactive protein, Troponin $\mathrm{T}$, paracetamol and salicylate, full blood count and D-Dimer test. 1065 patients were studied using equipment producing results comparable to those reported by the central laboratory. Compared to the main laboratory, the time to haematology results decreased by 47.2 minutes; to D-dimer results by 66.1 minutes and to chemistry by 41.3 minutes. The time to decide whether to discharge patients was reduced by 28.2 minutes but unfortunately the effect on patient waiting time was not reported.

Patient waiting times in A\&E depends on many factors including overall department efficiency, high demand for services (flu season; public holidays when GP practices), insufficient in-patient beds to accept referrals from $A \& E$, delays accessing specialist opinions, insufficient nursing or junior medical staff as well as delayed access to diagnostic services. These varied causes indicates that diagnostic services is one of many rate limiting steps determining patient waiting times in A\&E and that other approaches (e.g. time to clinical decision making) might more sensitively reflect the usefulness of POCT.

\section{Baseline measurement}

The time taken to produce POCT tests was calculated as the time elapse (TE) between requesting tests and the time at which completed results were returned to the ICE system. Patient waiting times were derived from the patient administration system (Symphony) used in the A\&E department. To assess the true effect of POCT on patient waiting times the analysis was confined to cases associated with POCT tests only $(n=217)$. A control population $(n=229)$ was randomly selected from the clinical laboratory database. The time interval between requesting a test and receiving the results for the POCT tests was 23 minutes and for the laboratory tests, 60 minutes. The patient waiting times (time of discharge âu time of arrival) was 167 minutes for the POCT group and 208 for the clinical laboratory group, a difference of 31 minutes.

The project confirmed that interfaced (on-line) POCT has many advantages with respect to the quality and safety of data management. The benefits include reliable identification of the requesting clinician and patient and the assurance that the right result is allocated to the right patient. The study also showed that the quality of results produced by the POCT devices met the requirements of the clinical environment.

\section{Design}

In this initiative, Two POCT devices were interfaced to a clinical electronic order communications system (ICE) via the hospital N3 network. The POCT devices included the Abbott I-Stat and the Sysmex XS haematology analyser. The I-Stat assayed serum concentrations of sodium, potassium, chloride, urea and creatinine (abbreviation - 'U\&E'). The XS produced a full blood count (FBC) including five part differential and messages describing pathological features requiring follow-up by microscopic examination. The I-Stat and XS were unidirectionally interfaced to a small server (Midlynx) which via the hospital network was linked to the ICE server.

Clinicians requisitioned test profiles using specially designed forms on the ICE system. Orders were downloaded to a networked printer which produced bar code labels that were read by the POCT devices. Completed test results were electronically transferred to the ICE system for viewing using networked terminals throughout the hospital. A comprehensive quality management system involved formal user training, internal and external QC checks and correlation with analysers located in the main clinical laboratory. The POCT devices were operated in shifts by two University students using material generated by the study to apply for the Msc degree by research rather than by examination. Data for the study was extracted from the hospital clinical systems using SQL queries.

\section{Strategy}

Considerable interest generated within the Trust. One possibility discussing whether portals of entry into the hospital should be 
served by POCT devices linked to the hospital network. Entry points under consideration being A\&E, AAU and UCC. The intention is to place the same POCT devices and test menus in each location to standardise rapid testing facilitating the hospital admissions process. The test repertoire included UE, FBC, Amylase, LFT, glucose, CRP and troponin I.

A reduction in waiting time was only observed when patients received POCT exclusively. Implementing this strategy involves multidisciplinary collaboration. A POCT policy document from senior management endorsed by the Trust Board and translated into SOPâCDs is used for training users to ensure appropriate and safe use of tests. In this regard, preliminary work should ensure POCT device test profiles and main laboratory demonstrate a degree of correlation satisfactory for the clinical environment thus increasing confidence in the POCT system encouraging clinicians to utilise the system for patient management.

Our study showed on-line POCT configuration was robust, reliable and user friendly, resolving staffing arrangement issues. HCAâDLs with appropriate training from the clinical laboratory produce accurate and precise results. Using on-line POCT devices at portals of entry to the hospital help front line staff accelerate decision making, reduce congestion and most importantly, improve patient waiting times. A comprehensive quality management system involved formal user training, internal and external QC checks and correlation with analysers located in the main clinical laboratory. The initiative is of significant interest to other healthcare providers especially those developing new patient care pathways. An important example is the proliferation of Urgent Care Centres (UCC) which is designed to reduce the number of less urgent cases referred to A\&E departments. In this context, the speed and efficiency of the clinical process will be enhanced by the rapid but safe delivery of pathology test results from on-line POCT services. Bearing in mind that ICE is highly prevalent throughout the NHS (approximately 200 placements), a huge trained user group is available to take advantage of on-line POCT services. Also, of significant interest to community polyclinics and large GP practices in the community. The results of this project were presented at a sponsored meeting on the 2nd July 2012. Hopefully further dissemination will follow though the usual channels including -presentations, posters and publication.

\section{Results}

The study showed that patients receiving test profiles from the POCT devices experienced a 31 minute reduction in waiting time compared to a control group receiving the same test profiles from the main laboratory. Waiting time was defined as the time elapse between patient registration to and discharge from the A\&E department. The time to produce test results (turnaround time) was 24 minutes for the POCT devices and 60 minutes for the equivalent laboratory tests (FBC; sodium, potassium, chloride, urea and creatinine). Turnaround time was defined as the time elapse between submitting a request on ICE to receiving all test results on ICE. The A\&E clinicians stated that compared to the conventional laboratory service, the rapid availability of POCT test results facilitated patient care in several respects:
1) Rapid, safe discharge of patients with less serious complaints 2) Very rapid management of patients with sepsis. Where cases caused concern, the proximity of the POCT devices was exploited with delivery of a full blood count within 3 minutes of delivery of the blood sample. This produced the necessary information to confirm the clinical diagnosis and institute antibiotic treatment without delay. 3) Similarly, the management of patients presenting with dangerous cardiac arrhythmias was expedited by the rapid (three minute delay) availability of serum potassium results.

\section{Lessons and limitations}

Communications with the supplier in the USA, the BT supplier, the lead Clinicians and hospital staff was deemed effective including the resolution of interface and HL7 messaging issues by the ICE and Midlynx suppliers. The work performed by both suppliers and the funding body NHS London being highly commendable. Another success being the testing of POCT devices in terms of speed of operation and accuracy of results. Additional training support was maintained by Quest diagnostics staff and suppliers regarding use of the Sysmex and Abbott analysers.

Limitations to the project included the time in obtaining the correct IP address information for the Hospital N3 network so firewall changes could be made requiring Liaison with suppliers at all levels. Also, the length of time taken for IT staff to address network and project issues and the length of time taken for ICE supplier to address interface and project issues could have fared better which included a lack of support from the ICE supplier in terms of some requested tasks. There was a definite lack of availability and cooperation of some clinical staff and engaging the Lead Clinicians was a major hurdle that we managed to eventually overcome. The initiative was supported by a grant from the NHS London Innovation Fund. A significant amount unspent due to unexpected levels of support received from industry partners.

The initiative not designed to demonstrate savings but to ascertain whether on-line POCT can contribute safely and reliably to the clinical process in a demanding environment such as an A\&E department. As part of these objectives, a financial estimate was carried out (5 year 'whole life approach') showing that the cost of a FBC produced by the Sysmex XS was close to that observed for the main laboratory while the I-Stat profile was more expensive than the laboratory equivalent. The financial model not taking into account savings that might accrue from the reduction in waiting times leading to generation of income through an increase in the number of managed clinical cases. Further work in this area is therefore required to assist decision makers choose whether to invest in on-line POCT services. Sophisticated modelling techniques are needed to balance the demonstrable clinical advantages against the costs involved. This analyses also needs to take into account the rapid evolution of POCT technology. Devices are becoming smaller, more accurate and safer to use with a test repertoire expanding into most clinical areas. Standards (ISO22870) covering the operational aspects of POCT, but the evaluation of benefit although intuitively obvious, needs assessment. 


\section{Conclusion}

The main objectives of the study were realized in firstly, establishing an âDDon-lineâCu configuration of validated POCT devices in the A\&E department and subsequently linking these devices to an electronic order communications system (ICE) allowing electronic test requesting and reporting which was the innovative aspect of the study. Generally, where POCT devices are interfaced, the connection is usually only between POCT and a laboratory computer system. Data capture enabled accurate measurement of the effect of on-line POCT on patient waiting times.

On-line POCT is a safe, effective and robust method of operating diagnostic equipment located remotely from the clinical laboratory.Interfacing POCT devices overcomes the main disadvantage (potentially unsafe clinical data handling) of deploying 'standalone' analytical equipment. The quality of POCT generated results is suitable for the clinical environment and this case study shows that POCT, when focused on a discrete group of patients, can be very advantageous in reducing patient waiting times significantly when a small repertoire of diagnostic tests is required for the management of cases in an A\&E department. Online POCT is thus cost effective, improves the patient experience and reduces patient waiting times in well defined circumstances, for example, patients with Sepsis to enable rapid diagnosis and insitution of treatment without delay, the management of patients presenting with dangerous arrythmias expedited by the rapid ( 3 minute) availability of serum potassium results, the safe and rapid discharge of patients with less serious complaints and is also useful in specialist clinics e.g. an anti-coagulation clinic.

\section{References}

1) Lee-Lewandrowski E, Corboy D, Lewandrowski K, Sinclair J, McDermot S, Benzer TI. Implementation of a point-of-care satellite laboratory in the emergency department of an academic medical center. Impact on test turnaround time and patient emergency department length of stay. Arch Pathol Lab Med. 2003 Apr; 127(4):456-60

2) Murray RP, Leroux M, Sabga E, Palatnick W, Ludwig L. Effect of point of care testing on length of stay in an adult emergency department.

J Emerg Med. 1999 Sep-Oct; 17(5):811-4.

3) Parvin CA, Lo SF, Deuser SM, Weaver LG, Lewis LM, Scott MG. Impact of point-of-care testing on patients' length of stay in a large emergency department. Clin Chem. 1996 May; 42(5):711-7.

4) Kendall J, Reeves B, Clancy M.Point of care testing: randomised controlled trial of clinical outcome.BMJ. 1998 Apr 4;

316(7137):1052-7.

5) Leman $P$, Guthrie D, Simpson R, Little F. Improving access to diagnostics: an evaluation of a satellite laboratory service in the emergency department. Emerg Med J 2004:21:452 - 456.
6) CLSI Document POCT1-A2. Clinical Laboratory Standards Institute (CLSI) Point of Care Connectivity; Approved Standard âDu

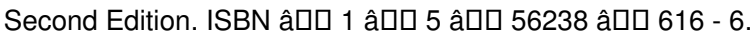

\section{Declaration of interests}

Nothing to declare

\section{Acknowledgements}

Misha Piracha - MSc student

Ladan Jama - MSc student 